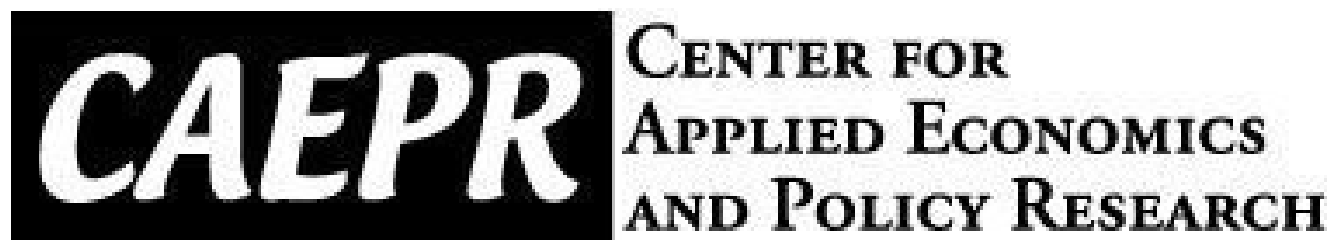

\author{
CAEPR \\ Working Paper \\ \#2018-008
}

\author{
Recursive Utility and Thompson Aggregators, II: \\ Uniqueness of the Recursive Utility Representation
}

\author{
Bob Becker \\ Indiana University \\ Juan Pablo Rincon- \\ Zapaterio \\ Univesidad Carlos III \\ de Madrid
}

August 10, 2018

This paper can be downloaded without charge from the Social Science Research Network electronic library at https://papers.ssrn.com/sol3/abstract_id=3239606

The Center for Applied Economics and Policy Research resides in the Department of Economics at Indiana University Bloomington. CAEPR can be found on the Internet at: http://www.indiana.edu/ caepr. CAEPR can be reached via email at caepr@indiana.edu or via phone at 812-855-4050.

(C)2018 by Bob Becker and Juan Pablo Rincon-Zapaterio. All rights reserved. Short sections of text, not to exceed two paragraphs, may be quoted without explicit permission provided that full credit, including (C) notice, is given to the source. 


\title{
Recursive Utility and Thompson Aggregators, II: Uniqueness of the Recursive Utility Representation*
}

\author{
Robert A. Becker \\ Department of Economics \\ Indiana University \\ Bloomington, IN 47405 \\ USA
}

\author{
Juan Pablo Rincón-Zapatero \\ Departamento de Economia \\ Universidad Carlos III de Madrid \\ 28903 Getafe (Madrid) \\ Spain
}

August 10, 2018

\begin{abstract}
We reconsider the theory of Thompson aggregators proposed by Marinacci and Montrucchio [30]. We demonstrate the Koopmans equation has a unique utility function solution given a Thompson aggregator. Uniqueness holds only on the interior of the commodity space's positive cone. Our proof verifies the Koopmans operator is a $u_{0}-$ concave operator. We verify this using general sufficient conditions due to Liang, et al [28]. Previous published results apply variants of the contraction mapping theorem to the space of possibly utility functions endowed with the Thompson metric. Concave operator methods work on the possible utility function space with its norm topology. Our approach combines order and metric structures to demonstrate uniqueness differently than in the existing literature.

JEL Codes: D10, D15, D50,E21

Keywords: Recursive Utility, Thompson Aggregators, Koopmans Equation, $u_{0}$ - Concave Operator Theory
\end{abstract}

\footnotetext{
*Corresponding author RAB (email: becker@indiana.edu). JPRZ emal: (email: jrincon@eco.uc3m.es). We thank the participants at the European Workshop in General Equilibrium Theory (Univeristy of Glasgow, June 2016) for their helpful comments on the uniqueness theory presented here and first appearing in our working paper [8]. Particular thanks go to Łukasz Balbus, Gaetano Bloise, Yiannis Vailakis, and Łukasz Woźny for helpful conversations and suggestions. Kevin Reffett deserves a special acknowledgment for his comments. RAB thanks him for all the conversations over the years about order structures and recursive methods. The usual caveat applies.
} 


\section{Introduction}

Recursive utility functions defined for discrete time, deterministic, and infinite horizon intertemporal choice problems have been studied intensively since their introduction by Koopmans ([20], [21], and [22]). Koopmans, Diamond and Williamson [23] extended that research. Koopmans proved a recursive utility function satisfied a particular functional equation, known today as the Koopmans equation. This equation relates the utility function to an aggregator function in two real variables, current consumption and future utility.

Lucas and Stokey [29] proposed taking the aggregator as the primitive concept. Using that function, the Koopmans equation in the unknown utility function is defined and a unique solution (in an appropriate function space) is sought. This solution recovers a unique recursive utility function representation of the underlying preference relation defined on the commodity space. This existence and uniqueness problem is solved by setting up a fixed point problem for the Koopmans operator. It is a selfmap defined on the given space of potential utility functions. They appeal to Banach's Contraction Mapping Principle. This yields the existence and uniqueness of the Koopmans equation's solution. In order to do so, the aggregator function must be carefully restricted in order to prove the Koopmans operator satisfies Blackwell's [11] sufficient conditions for a contraction mapping. Blackwell's sufficient conditions include the requirement that the given transformation be a monotone operator. Generalizations of their approach are the subject of Boyd [13] and the monograph by Becker and Boyd [6]. Their results are confined to the class of Blackwell aggregators. ${ }^{1}$

Marinacci and Montrucchio [30] introduce the Thompson class of aggregators. Many Thompson examples fail to be members of the Blackwell class. Contraction mapping methods based on verification of Blackwell's sufficient conditions do not apply. However, the Koopmans operator is a monotone operator in both the Blackwell and Thompson aggregator cases. They separate the existence and uniqueness results by first proving existence by appealing to Tarski's Fixed Point Theorem [38], and separately linking uniqueness to showing the Koopmans operator (on a restricted commodity space domain) is a contraction mapping when the admissible space of possible utility functions is assigned the Thompson metric. ${ }^{2}$

We reexamine Marinacci and Montrucchio's [30] existence result in Becker and Rincón-Zapatero [9]. We also demonstrate the existence of extremal fixed points for the Koopmans operator. That is, there are least (and greatest) fixed points. We go beyond their existence theory using order continuity arguments justify approximations of the least (greatest) fixed point from below (above). These approximations are linked to qualitative properties (e.g. monotonicity, concavity, and norm semicontinuity) of the extremal solutions. Neither our methods nor those in Marinacci and Montrucchio [30], based on monotone op-

\footnotetext{
${ }^{1}$ Subsequent research on Blackwell aggregators include Le Van and Vailakis [27], Martinsda-Rocha and Vailakis ([32], [33]), and Rincón-Zapatero and Rodriguez-Palmero ([36], [37].

${ }^{2}$ The operator is shown to be a contraction by a direct argument constructing the required Lipschitz constant.
} 
erator theory alone, are sufficient for an adequate uniqueness theory.

The purpose of our paper is to prove a uniqueness result employing concave operator theory. ${ }^{3}$ Towards this end, we must restrict the underlying commodity space in two ways. First, we do not admit sustained or balanced growth paths for consumption streams over the infinite horizon (discrete time). This restriction is modeled by assuming the commodity space is the space of all bounded nonnegative-valued sequences. It is the positive cone of the space of all bounded real-valued sequences. The second constrains commodity sequences to the norm interior of the commodity space. Restrictions of the latter type are noted in Marinacci and Montrucchio [30] and Martins-da-Rocha, Vailakis [32], and Bloise and Vailakis [12]. The former paper admits sustainable growth, whereas the latter two articles rule out sustainable growth. Martins-da-Rocha, Vailakis [32], and Bloise and Vailakis [12] confine the commodity sequences to the interior of the commodity space's positive cone and we do this as well. ${ }^{4}$ Marinacci and Montrucchio [30] and Martins-da-Rocha and Vailakis [32] use Thompson metrics to define a distance in their underlying space of possible utility functions. The former uses the contraction mapping theorem directly whereas the latter exploits local contraction theory as an application of their new mathematical fixed point results. ${ }^{5}$ We retain the original norm topology for the space of possible utility functions. Critical mathematical properties important for a concave operator theory application follow (e.g. the positive cone is closed and has a nonempty norm interior).

Marinacci and Montrucchio [31] present a uniqueness theorem for Thompson aggregators that is valid on the interior of the commodity space's positive cone. That work is independent of ours; it applies to their original axiom list defining the Thompson class. We strengthen their defining conditions by assuming the aggregator is jointly continuous and concave in its arguments. Their uniqueness proof imposes the Thompson metric on the space of possible utility functions. This yields both existence and uniqueness of the recursive utility function belonging to a given Thompson aggregator. We draw on our general existence theory for extremal solutions to the Koopmans equation in proving that equation has is a unique solution.

Marinacci and Montrucchio [31] base their results on a careful order theoretic description of the boundary of both the utility function and commodity spaces. ${ }^{6}$ We emphasize the topological features of the positive cone's boundary relative to the positive cone as well as the conditions characterizing interior points in the positive cone. The sup-norm topology is maintained throughout

\footnotetext{
${ }^{3}$ Our first working paper, Becker and Rincón-Zapatero [8], combines our uniqueness and existence theories in a single article. The present paper is based on the uniqueness section, but differs in some fine details.

${ }^{4}$ We characterize the sequences in the positive cone's norm interior as well as those in its boundary. The latter consumption sequences are where uniqueness can fail.

${ }^{5}$ See Rincón-Zapatero and Rodriguez-Palmero ([36], [37]) for the introduction of local contraction theory in recursive economic models.

${ }^{6}$ They link their order theoretic description to a topological description for their applications. We simply go to the topological consideration at the outset as this is what is actually used to obtain the uniqueness result by $u_{0}-$ concave operator methods.
} 
and its properties are critical. For example, the positive cone is automatically norm closed in our setup. We combine order theoretic ideas and topological considerations by observing our function spaces are abstract AM-spaces with units. $^{7}$ This linkage between order and topology (via the norm topology) is a central structural framework and critical for our mathematical approach to the uniqueness question.

The novel feature of our uniqueness theory is its reliance on $u_{0}$ - concave operator theory pioneered by Krasnosel'skiu [24]. ${ }^{8}$ Coleman [14] introduced this technique in the economics literature as a tool for proving a unique equilibrium exists in a dynamic equilibrium model of capital accumulation (subject to capital taxation).${ }^{9}$ He does not quote Krasnosel'skiı̌'s Theorem [24] directly. Rather, he uses it to inspire a different variation that is better adapted to his problem. We directly quote Krasnosel'skiı's Theorem [24]. However, verifying his conditions for the Koopmans operator relies on relatively recent work by Liang, et al [28] who provide verifiable conditions for the Koopmans operator problem that are sufficient for application of $u_{0}$ - concave operator theory. Restrictions on the commodity space play an important role in checking their sufficient conditions in our setup.

Section 2 offers a brief review of concepts on partially ordered sets, lattices, positive cones in real Banach spaces, and a review of basic concave operator theory. Thompson aggregators and recursive utility functions are developed in Section 3. The general impossibility of uniquely determining a recursive utility function from a given Thompson aggregator is addressed by means of a counterexample. The minimal and maximal consumption sequence restrictions necessary for a positive unique determination of the recursive utility from the given Thompson aggregator is presented in Section 4. Verification that the Koopmans operator is a $u_{0}$-concave operator is found in Section 5 . Concluding comments follow.

\section{Mathematical Structures}

The uniqueness theory presented in this paper combines order and metric properties of the commodity and utility function vector spaces. Important mathematical structures critical for our uniqueness theory are developed below.

\footnotetext{
${ }^{7}$ See Section 2.2 for background on these spaces and special features of their positive cones.

${ }^{8}$ We actually use the form of Krasnosel'skiî's Theorem [24] presented in Krasnosel'skiı̌ and Zabreǐko [25]. They recast the result in a more general form than the nonlinear eigenvalue problem motivating Krasnosel'skiı's seminal work.

${ }^{9}$ Datta and Reffett [18] survey monotone operator methods in recursive models and include uniqueness arguments for Coleman's model based on $u_{0}$ - concave operator methods. Le Van, Morhaim, and Vailakis [26] prove a version of Krasnosel'ski1's Theorem that also includes a monotone operator existence result. They use their result to prove a unique solution exists in a determinstic dynamic programming setup.
} 


\subsection{Positive Cones and Nonlinear Operators in Riesz Spaces}

A set $X$ is said to be partially ordered, or a poset, if it is nonempty and for certain pairs $(x, y)$ in $X \times X$ there is a binary relation $x \leq y$ which is reflexive, transitive, and antiysmmetric. ${ }^{10} \mathrm{~A}$ poset $X$ is a lattice provided each pair of elements has a supremum (sup, meet) and an infimum (inf, join). Standard lattice notation for sups and infs is followed: $\sup \{x, y\}=x \vee y$ and $\inf \{x, y\}=x \wedge y$. A complete lattice is a lattice in which each nonempty subset $Y$ has a supremum $\bigvee Y$ and an infimum $\bigwedge Y$.

Let $E$ denote a real vector space. The zero element in $E$ is denoted by $\theta$. A nonempty subset $P$ of $E$ is said to be a cone if (i) $P+P \subseteq P,(i i) \lambda P \subseteq P$ for each scalar $\lambda \geq 0$, (iii) $P \cap(-P)=\{\theta\}$. We note that a cone as defined here is also a convex set and is said to be a pointed convex cone, or more simply, a convex cone. ${ }^{11}$ A cone induces a partial order on the vectors belonging to $E$. A vector $x$ is said to be positive, written $x \geq \theta$, provided $x \in P$. The cone is then called the positive cone of $E$ and is denoted by $E^{+}$in the sequel. We use the notation $P$ to denote an arbitrary cone (which may not be the positive cone). The standard partial relation expressing $x \geq y$ whenever $x, y \in E$ is defined by requiring $x-y \in E^{+}$. Write $x>\theta$ whenever $x \geq \theta$ and $x \neq \theta$. Likewise, $x>y$ provided $x \geq y$ and $x \neq y$.

Our application requires the vector spaces are Riesz spaces where $E$ is equipped with the partial order derived from the cone $E^{+}$. A Riesz space is a partially ordered vector space that is also a lattice. ${ }^{12}$ For each element $x \in E$, we define its positive part, $x^{+}$, its negative part $x^{-}$, and its absolute value, $|x|$, by the formulas:

$$
x^{+}=x \vee \theta, x^{-}=x \wedge \theta \text {, and }|x|=x \vee(-x) .
$$

An order interval in the Riesz space $E$ is a set of the form $\langle x, y\rangle=$ $\{z \in E: x \leq z \leq y\}$. A subset $G$ of a Riesz space is order bounded from above if there is a $y \in E$ such that $z \leq y$ for each $z \in G$. The dual notion that this subset is order bounded from below is defined similarly. A subset of a Riesz space is order bounded if it is contained in an order interval. $E$ is order complete, or Dedekind complete, if every nonempty subset that is order bounded from above has a supremum (and dually, every nonempty subset that is order bounded from below has an infimum).

Suppose $E$ is also a real Banach space. There are additional restrictions on that Banach space's positive cone required for the application of concave operator theory. For example, the cone must be closed in the norm topology. This condition is met by positive cones of the spaces appearing in our setup.

\footnotetext{
${ }^{10}$ Birkhoff [10] and Davey and Priesley [15] cover the basic properties of posets and lattices.

${ }^{11}$ Our definition follows Aliprantis and Tourky [4]. They note that definitions of cones may vary among authors.

${ }^{12}$ Riesz spaces are also known as vector lattices. Consult Aliprantis and Border ([1], Chapter 8) for a thorough review of Riesz spaces. We follow their terminology. All Riesz spaces appearing in our paper are Archimedean.
} 
The notation $x>>\theta$ means $x \in \operatorname{int}\left(E^{+}\right) \equiv E^{++}$, where $\operatorname{int}\left(E^{+}\right)$denotes the norm interior of the cone $E^{+}$. Of course, this latter inequality is only meaningful when $\operatorname{int}\left(E^{+}\right) \neq \varnothing-$ a strong topological restriction on the underlying Banach space. An arbitrary cone $P$ contained in $E$ with nonempty interior in its norm topology is said to be a solid cone. The positive cones are solid in our applications.

The positive cone $E^{+}$is called normal if there is a constant $\mathcal{N}>0$ such that $0 \leq x \leq y$ implies $\|x\| \leq \mathcal{N}\|y\|$ and $\mathcal{N}$ does not depend on the choices of $x$ and $y$. In our application the norm constant $\mathcal{N}=1$.

We consider an abstract nonlinear operator, denoted by $A$, that is positive on $E^{+}$. That is, it is a self-map: $A: E^{+} \rightarrow E^{+}$. We also write this as $A E^{+} \subseteq E^{+}$. The operator $A$ is said to be monotone (isotone, increasing) on $E^{+}$if $x \leq y,\left(x, y \in E^{+}\right)$implies $A x \leq A y$. The Koopmans operator derived from a Thompson aggregator is monotone.

Given a nonlinear operator satisfying $A E^{+} \subseteq E^{+}$we are concerned with the existence of fixed points as well as whether or not there is a unique solution in the cone $E^{+}$. The operator equation is $A x=x$ with $x \in E^{+}$; a solution is a fixed point of the operator, $A$. In some applications there may be a trivial fixed point, $\theta$. We are only interested in nontrivial fixed points $x \in E^{+}$with $x \neq \theta$. The Koopmans operator does not admit a trivial fixed point under our assumptions.

All spaces appearing in this paper are complete normed Riesz spaces. They are also Banach lattices. That is, they are Riesz spaces which are Banach spaces whose norms are also lattice norms. ${ }^{13}$ A norm $\|\bullet\|$ on a Riesz space is a lattice norm provided for each point $x$ and $y,|x| \leq|y|$ implies $\|x\| \leq\|y\|$. The corresponding positive cone is clearly normal.

The spaces on which the Koopmans operator acts turn out to be abstract $M$ - spaces, or AM-spaces with an order unit. AM-spaces are Banach lattices for which $\|x \vee y\|=\max \left\{\|x\|,\|y\|\right.$ for each $\left.x, y \in E^{+}\right\}$. An $A M-$ space $E$ possesses an order unit whenever there exists an element $e \in E$, $e>\theta$, such that for each $x \in E$ there is a scalar $\lambda>0$ satisfying $|x| \leq \lambda e$. If an $A M-$ space has a unit, then its lattice norm is defined for each $x \in E$ by $\|x\|_{\infty}=\inf \{\lambda>0:|x| \leq \lambda e\}$. This norm is equivalent to the given norm on $E$. One advantage to this setup is that the positive cone of an $A M-$ space with unit is norm-closed, convex, solid and normal. ${ }^{14}$

\footnotetext{
${ }^{13}$ See Aliprantis and Border [1], Aliprantis and Burkinshaw [2], Meyer-Nieberg [34], Peressini [35], and Vulikh [39] for details on Riesz spaces and Banach lattices.

${ }^{14}$ The positive cone of an AM-space has a nonempty norm interior provided it is an AMspace with unit. See Aliprantis and Tourky ([4], p. 65) and Peressini ([35], p. 183). A Banach lattice has an order unit if and only if that order unit is an interior point of the space's positive cone. In this case, the original sup norm and lattice norm topologies are equivalent. See Meyer-Nieberg ([34], Corollary 1.2.14 for details).
} 


\section{$2.2 u_{0}-$ Concave Operators and Uniqueness Theory for Nonlinear Operators}

The Koopmans operator belonging to a Thompson aggregator is a self-map on the positive cone of a real Banach space. Properties of this cone play a critical role in our uniqueness theory. We review some basic uniqueness theory for an abstract nonlinear operator defined on an arbitrary norm-closed convex cone $P$ of a real Banach space.

Our purpose is to apply results obtained for uniqueness of solutions without relying on a (generalized) contraction mapping theorem, but instead drawing on features derived from the presence of a partial order on elements of the underlying real Banach space. We apply $u_{0}$ - concave operator theory designed to promise at most one solution in the nonempty, closed, and pointed convex cone $P$ as originally developed by Krasnosel'skiı̌ [24] and later exposited in Krasnosel'skiı̌ and Zabreǐko [25].

Our exposition draws on the presentation by Krasnosel'skiı̌ and Zabreǐko [25] and Krasnosel'skiı [24]. The latter reference introduces the class of $u_{0}-$ concave operators. We present new results obtained by Liang, Wang, and Li (hereafter, Liang et al) [28]. Closely related references include Guo and Lakshmikantham [16] and Guo, Cho, and Zhu [17].

\subsubsection{The Krasnosel'skiı̌ and Zabreǐko Theorem}

Fix a non-zero element, denoted by $u_{0}$, in a cone $P$ contained in a Banach space. Maintain the assumption that this cone is nonempty, norm-closed, and convex. Refer to $P$ as the cone $P$ in our general mathematical overview. Our applications are made to the positive cones in real Banach spaces.

Let $\theta$ denote the zero element in $P$. An operator $A: P \rightarrow P$ is called $\mathbf{u}_{0}$-concave on $P$ if for each non-zero element $x \in P$ there are positive scalars $a(x)$ and $b(x)$ such that

$$
a(x) u_{0} \leq A x \leq b(x) u_{0}
$$

and if for each $x \in P$ with $a_{1}(x) u_{0} \leq x \leq b_{1}(x) u_{0}$, for some $a_{1}, b_{1}>0$, we have

$$
A(t x) \geq[1+\eta(t, x)] t A x \text { for } 0<t<1,
$$

where $\eta(t, x)>0$.

The restrictions $a_{1}(x) u_{0} \leq x \leq b_{1}(x) u_{0}$ and $u_{0} \neq \theta$ taken together mean that $x>\theta$, but not necessarily that $x>>\theta$. Inequality (2) implies $A(t x)>t A x$ holds. This falls short of asserting $A(t x)>>t A x$ - that is, the vector difference $A(t x)-t A x \in \operatorname{int}(P)$. This interiority property cannot even be considered unless the underlying cone is solid. ${ }^{15}$ Hence, we work with the inequalities expressed in (2). This turns out to be sufficient for settling our uniqueness question.

\footnotetext{
${ }^{15}$ It happens that our cones are solid. But, the general mathematical theory does not impose that particular requirement.
} 
The main Krasnosel'skiı̌ and Zabreiko result ([25], Theorem 46.1, p. 290) is stated below. ${ }^{16}$

Theorem 1 (Krasnosel'ski and Zabreǐko). Let $A$ be a monotone operator which is $u_{0}-$ concave on $P$. Then the equation

$$
A x=x
$$

has at most one non-zero solution in $P$.

The result emphasizes order theoretic ideas based on the partial ordering of the underlying function space as defined by the elements of the cone $P$. Topological considerations enter through the underlying Banach space structure (which explicitly determines the norm-topology) whereby $P$ is a closed set. It is of some interest to note that this result does not require the cone $P$ to be a normal cone. However, the positive cones are normal in our setup.

Krasnosel'skiǔ and Zabrěko's Theorem imposes two conditions on the operator $A$ - it is monotone and $u_{0}$-concave. The Koopmans operator is monotone, so the only task in applying their theorem in our setting concerns verification that it is a $u_{0}-$ concave operator.

There are two critical estimates that must be satisfied to verify $A$ is a $u_{0}-$ concave operator in the general abstract setting. The method of Liang et al [28] offers a set of sufficient conditions that, once (1) is verified, implies (2) is also satisfied. A second type of concave operator can prove useful in verifying the $u_{0}$-concave property. Liang et al's ([28]) contribution turns on using this second idea about expressing concavity for abstract operators.

\subsubsection{The Liang, Wang and Li Ordered Concave Operator Theorem}

Fix the function $u_{0}>\theta$ with $u_{0} \in P$ as above. Define

$$
P_{u_{0}}=\left\{x \in P: \exists a(x), b(x)>0 \text { such that } a(x) u_{0} \leq x \leq b(x) u_{0}\right\} .
$$

Clearly $P_{u_{0}} \subset P$. The set $P_{h_{0}}$ is readily seen to be a convex set.

Let $P^{\prime}=\operatorname{range}(A) \subseteq P$ denote the range of the operator $A$ acting on the cone $P$. This range set $P^{\prime}$ may also be a cone, but this is not a formal requirement. What matters is that $P^{\prime}$ inherits the partial order induced by the cone, $P$ as well as be a convex subset of $P$.

Condition (1) differs from (3). The former uses the image of $x$ under the mapping $A$, whereas the latter employs the domain point, $x$. The set $P_{u_{0}}$ is the operator's "target." This is why we must establish (1) in order to verify $A$ has the $u_{0}$ - concavity property.

Definition $2 A: P \rightarrow P^{\prime}$ is an ordered concave operator if for each $x, y \in$ $P$ with $x \geq y$ and $t \in[0,1]$ :

$$
A(t x+(1-t) y) \geq t A x+(1-t) A y .
$$

\footnotetext{
${ }^{16}$ Krasnosel'skir ([24], p. 188) presents the first formulation of this result within the context of nonlinear eigenvalue problems.
} 
The convexity of the domain and range spaces is critical for the inequality above to make sense. The former set is convex since the cone is a convex set. The latter is an assumption that must be checked in applications.

An ordered concave operator need NOT automatically be a $u_{0}$ - concave operator. Some authors (e.g. [16] and [17]) identify the terms concave and ordered concave operators. We prefer to keep the prefix "ordered" in place as a reminder the condition $x \geq y$ has an important role to play in this theory.

The following lemma is crucial. It is implicit in Liang et al's proof ([28], Lemma 4, p. 579). We note that $P_{u_{0}} \subset P$.

Lemma 3 Suppose $A: P \rightarrow P_{u_{0}}$. Then for each $x \in P$ there is a positive number $\mu(x)$ such that

$$
A \theta \geq \mu(x) A x .
$$

Proof. Let $x \in P$. Then $A x \in P_{u_{0}}$ by assumption. By the definition of $P_{u_{0}}$ there are numbers $a(A x)>0, b(A x)>0$ such that

$$
a(A x) u_{0} \leq A x \leq b(A x) u_{0} .
$$

In particular, $x=\theta \in P$. Thus, the above inequality for $\theta$ is also true and reads

$$
a(A \theta) u_{0} \leq A \theta \leq b(A \theta) u_{0} .
$$

Combining both sets of inequalities as follows we have

$$
\begin{aligned}
A \theta & \geq a(A \theta) u_{0}=\left(\frac{a(A \theta)}{b(A x)}\right) b(A x) u_{0} \\
& \geq \mu(x) A x,
\end{aligned}
$$

where

$$
\mu(x) \equiv\left(\frac{a(A \theta)}{b(A x)}\right)>0
$$

We notice that the function $\mu(x)$ depends on the fixed operator, $A$, and the zero function, $\theta$ as $x$ varies in $P$.

Theorem 4 (Liang et al [28]) Suppose $A: P \rightarrow P_{u_{0}}$ is an ordered concave operator. Then $A$ is a $u_{0}$ - concave operator. ${ }^{17}$

Proof. For each $0<t<1$ : the ordered concavity condition implies, since $x \geq \theta$, that

$$
\begin{aligned}
A(t x) & =A(t x+(1-t) \theta) \\
& \geq t A x+(1-t) A \theta \\
& \geq t A x+(1-t) \mu(x) A x(\text { by }(5)) \\
& =t\left(1+\frac{(1-t) \mu(x)}{t}\right) A x .
\end{aligned}
$$

\footnotetext{
${ }^{17}$ We include their proof in order to show how the previous lemma implies an ordered concave operator is also a $u_{0}$ - concave operator.
} 
Now set

$$
\eta(t, x)=\frac{(1-t) \mu(x)}{t}>0
$$

since $\mu(x)>0$. Hence, $A$ is a $u_{0}-$ concave operator as we have shown

$$
A(t x) \geq(1+\eta(t, x)) t A x .
$$

The practical impact of this result is plain - check that an operator is a $u_{0}$ - concave operator on the cone $P$ by verifying the sufficient condition in Liang, Wang, and Li's Theorem! This effectively means checking that the given operator satisfies condition (5). The application of $u_{0}-$ concave operator theory to the Koopmans operator, $T_{W}$, turns on checking that condition. Doing so means there must be a clear choice of the cone, $P$, selection of the point $u_{0}$, verification that $T_{W} U$ belongs to the corresponding set $P_{u_{0}}$ whenever $U \in P$, and checking $T_{W}$ is an ordered concave operator. Additional restrictions on the underlying commodity space must be imposed to show the Koopmans operator is a $u_{0}$-concave operator. We offer an economically motivated condition towards this end in Section 4.

\section{Recursive Utility Theory for The Thompson Aggregator Class}

We begin with the basic background structures defining Thompson aggregators. Our assumptions strengthen those in Marinacci and Montrucchio [30] by imposing joint concavity and continuity properties on the aggregator. We also explicitly list the subhomogeneity and limit conditions employed in their existence proof (and ours).

\subsection{Defining Properties of Thomson Aggregators}

The class of Thompson aggregators is delineated by the following four basic assumptions.

Definition $5 W: \mathbb{R}_{+}^{2} \rightarrow \mathbb{R}$ is said to be a concave Thompson aggregator if it satisfies properties (T1) - (T6):

(T1) $W \geq 0$, continuous, and monotone: $(x, y) \leq\left(x^{\prime}, y^{\prime}\right)$ implies $W(x, y) \leq$ $W\left(x^{\prime}, y^{\prime}\right)$.

(T2) $W(x, y)=y$ has at least one nonnegative solution for each $x \geq 0$.

(T3) $W$ is a concave function of $(x, y)$.

(T4) $W(x, 0)>0$ for each $x>0$. 
(T5) $W$ is $\gamma$ - subhomogeneous - there is some $\gamma>0$ such that:

$$
W\left(\mu^{\gamma} x, \mu y\right) \geq \mu W(x, y)
$$

for each $\mu \in(0,1]$ and each $(x, y) \in \mathbb{R}_{+}^{2}$. If the defining inequality in (T5) is an equality, then we say $W$ is $\gamma$-homogeneous.

(T6) $W$ satisfies the MM-Limit Condition: for a given $\alpha \geq 1$ and $\gamma>0$ (from $(T 5)$ ),

$$
\lim _{t \rightarrow \infty} \frac{W(1, t)}{t}<\alpha^{-1 / \gamma}
$$

with $t>0$.

Our definition of a concave Thompson aggregator builds in the assumption that it is jointly continuous and concave in $(x, y)$ over $\mathbb{R}_{+}^{2}$. This differs somewhat from the formal assumptions given in Marinacci and Montrucchio [30]. In the sequel understand the shortened expression Thompson aggregator refers to concave Thompson aggregators unless otherwise indicated.

Our strengthened axioms are appropriate for solving the existence and uniqueness problem for the Koopmans operator equation. Our maintained conditions are satisfied in the major examples of Thompson aggregators.

A stronger version of Assumption (T5) is met in some examples (see the CES case below). That is, the aggregator satisfies positive homogeneity of degree $\gamma$ provided

$$
W(\mu x, \mu y)=\mu^{\gamma} W(x, y)
$$

for each $\mu>0$ and each $(x, y) \in \mathbb{R}_{+}^{2}$.

The parameter $\alpha>0$ in (T6) is the economy's maximum possible consumption growth factor. Condition (T6) turns out to be an important joint restriction on the preferences embodied in the aggregator function as well as on the underlying commodity space, as might arise from properties of technologies in production economies and/or endowments in exchange economies. ${ }^{18} \mathrm{In}$ our setting the commodity space excludes sustainable growth and the parameter $\alpha=1$.

\subsection{Examples of Thompson Aggregators}

There are two important sources for examples. The KDW aggregator (defined below) has parameterizations placing it outside the Blackwell class and firmly in the Thompson family. There are also many new examples based on the functional form for utility functions and production functions commonly studied in microeconomic theory. Both the CES (Constant Elasticity of Substitution) and KDW (Koopmans, Diamond, and Williamson [23]) examples satisfy (T1) (T6) with $\alpha=1$.

\footnotetext{
${ }^{18}$ Becker and Boyd [6] cover many examples of models with commodity spaces arising in interemporal choice and consistent with our Thompson aggregator specification.
} 
The class of CES aggregators are defined parametrically by the formula:

$$
W(x, y)=(1-\beta) x^{\rho}+\beta y^{\rho}, \text { for } 0<\rho \leq 1 .
$$

The parameter $\beta$ is restricted $-0<\beta<1$. Note that this family of functions is positively homogeneous of degree $\rho$. The elasticity of substitution is $\sigma:=1 /(1-\rho) ; \rho \neq 1$. All six Thompson aggregator criteria are met when $\sigma>1$. Assume this restriction applies without further notice. The CES Thompson aggregators are readily shown to satisfy (T5) with $\gamma=1$.

Other Thompson aggregators are available as variations on the CES theme (see Marinacchi and Montrucchio [30]). For example, the quasi-linear aggregator $W(x, y)=x+\beta y^{\rho}$ is Thompson for the same parameter restrictions imposed on the CES family.

The KDW aggregator is defined by the formula

$$
W(x, y)=\frac{\delta}{d} \ln \left(1+a x^{b}+d y\right)
$$

where $a, b, d, \delta>0$. This is a Thompson aggregator that satisfies (T5) with $\gamma=b^{-1}$. It is a Blackwell aggregator when "discounting" is assumed using the restriction $\delta<1$. Similarly, "upcounting." or "no discounting," is permissible in the Thompson class. These cases correspond to $\delta>1$ and $\delta=1$, respectively.

\subsection{The Commodity Space}

The vector space $\ell_{\infty}$ is the set of all real-valued sequences endowed with the sup norm, $\|x\|=\sup _{t}\left|x_{t}\right|$, where $x=\left\{x_{1}, x_{2}, \ldots\right\}$ denotes a sequence. The commodity space is the positive cone denoted by $\ell_{\infty}^{+}$. This is a typical commodity space in deterministic optimal growth models where all feasible consumption paths are bounded given diminishing marginal returns in the model's production sector. There is a technical reason for choosing this commodity space - the order intervals capturing minimal and maximal consumption constraints must be shift invariant in the recursive utility framework. Marinacci and Montrucchio [30] use a different shift invariant domain that, unlike ours, accomodates growing economies. Our choice of domain is motivated by its intuitive economic basis and for its technical conveniance in demonstrating the Koopmans operator is a $u_{0}$ - concave operator.

A constant vector in $\ell_{\infty}$ is denoted by $x_{c o n}=\{x, x, x, \ldots\}$, where $x$ is a real number. The zero vector is a constant sequence of zeroes in each term. It is denoted by 0 . The constant vector $e=\{1,1,1, \ldots\}$ is an order unit and it is a norm-interior point of the positive cone, $\ell_{\infty}^{+}$. As $\ell_{\infty}$ is an AM-space with unit, it follows that the positive cone is closed, solid, and normal. It is readily verified that it is also a convex cone.

The positive cone's norm interior, denoted $\ell_{\infty}^{++}$, has a prominent place in uniqueness theories for Thompson aggregators. A sequence $x \in \ell_{\infty}^{+}$is an interior point of the positive cone (with the norm topology) if and only if it is 
an order unit. ${ }^{19}$ This implies the positive cone's norm interior is characterized as $\ell_{\infty}^{++}=\left\{x \in \ell_{\infty}^{+}: \inf _{t} x_{t}>0\right\}$. The boundary of the positive cone, denoted $\partial \ell_{\infty}^{+}$, is the complement of the interior relative to the positive cone. That is,

$$
\partial \ell_{\infty}^{+}=\ell_{\infty}^{+} \backslash \ell_{\infty}^{++}
$$

Since the positive cone is a normed closed subset of $\ell_{\infty}^{+}$and the cone's interior is necessarily open, it follows that the boundary is a norm closed set. Recall the notion of a boundary is a topological concept. ${ }^{20}$

A sequence $x \in \partial \ell_{\infty}^{+}$if and only if $\inf _{t} x_{t}=0$. A sequence belongs to $c_{0}$ if it converges to zero. A convergent sequence is also bounded, and hence an element of $\ell_{\infty}$. The vector space $c_{0}$ is also known as the space of null sequences. Its positive cone, $c_{0}^{+}$, is the corresponding set of nonnegative null sequences. Assign $c_{0}$ the sup - norm topology. It is an AM-space that contains no order units. ${ }^{21}$ Each point in $c_{0}^{+} \subset \ell_{\infty}^{+}$must be a boundary point in $\ell_{\infty}^{+}$. There are other boundary points. Any sequence in $\ell_{\infty}^{+}$with finitely many components equal to zero is also a boundary point. For instance, the sequence $\{0,1,1, \ldots\} \in \partial \ell_{\infty}^{+}$. Likewise, each sequence in $\ell_{\infty}^{+}$that has a convergent null subsequence (including the case of a subsequence of zeroes in each term) must be boundary points as well.

Consumption sequences in the commodity space are generally denoted by $C=\left\{c_{t}\right\}_{t=1}^{\infty}$; we write $C=\left\{c_{t}\right\}$ when the meaning is clear. Define the shift operator $S: \ell_{\infty}^{+} \rightarrow \ell_{\infty}^{+}$according to the rule $C=\left\{c_{1}, c_{2}, c_{3}, \ldots\right\} \mapsto S C=$ $\left\{c_{2}, c_{3}, \ldots\right\} .^{22}$ The definition of the shift operator says that the positive cone is invariant under its action: $S\left(\ell_{\infty}^{+}\right) \subseteq \ell_{\infty}^{+}$. There are other sets which are invariant with respect to the shift and they are important in our uniqueness theory. Evidently $\ell_{\infty}^{++}$is shift invariant. However, the boundary set, $\partial \ell_{\infty}^{+}$, is NOT shift invariant: $S(\{0,1,1, \ldots\})=\{1,1,1, \ldots\} \notin \partial \ell_{\infty}^{+}$.

\subsection{The Space of Possible Utility Functions}

Utility functions corresponding to a Thompson aggregator may be unbounded from above. We introduce a weighted space of possible utility functions in order to work within a space of suitably bounded real-valued functions.

First, define a weight function, $\varphi_{\gamma}$ following Marinacci and Montrucchio's [30] specification. For each $C \in \ell_{\infty}^{+}$define $\varphi_{\gamma}$ by the formula:

$$
\varphi_{\gamma}(C)=(1+\|C\|)^{1 / \gamma} .
$$

This weight function is uniformly continuous and convex on $\ell_{\infty}^{+}$with respect

\footnotetext{
${ }^{19}$ See Aliprantis and Tourky [4], p. 65) and Peressini ([35], p. 183).

${ }^{20}$ General details about the boundary concept in a topological space may be found in Kelley ([19], pp. 46-47).

${ }^{21}$ See Aliprantis and Border ([1], p. 529).

22 The shift operator can be defined as a self-map on the vector space $\ell_{\infty}$. However, the shift operator is invariant on that space's positive cone and this is the feature we utilize.
} 
to the sup - norm topology. ${ }^{23}$ Here, the parameter $\gamma>0$ appearing in the weight function is taken from $(T 5)$. This weight function as well the sup - norm entangle preference and technology parameters - the growth rate $\alpha=1$ is derived from a model's technology side while the parameter $\gamma$ comes from the model's preference side.

Definition 6 A function $U: \ell_{\infty}^{+} \rightarrow \mathbb{R}$ is $\varphi_{\gamma}-$ bounded provided

$$
\|U\|_{\gamma}:=\sup _{C \in \ell_{\infty}^{+}} \frac{|U(C)|}{(1+\|C\|)^{1 / \gamma}}<+\infty .
$$

The set of all $\varphi_{\gamma}-$ bounded real-valued functions with domain $\ell_{\infty}^{+}$is denoted by $B$. Its positive cone is $B^{+}$.

The zero function, $\theta$, is defined by $\theta(C)=0$ for each $C$. The zero function is the origin in the vector space $B$. The space $B$ is a Dedekind complete Riesz space with the pointwise partial ordering. Clearly the weight function $\varphi_{\gamma}$ satisfies $\varphi_{\gamma}(\theta)=1$ and $\varphi_{\gamma}(C) \geq 1$ for each $C$. Moreover, $\left\|\varphi_{\gamma}\right\|_{\gamma}=1$ as well and $\varphi_{\gamma}$ is an order unit in $B$. Hence, $B$ is an abstract $M$ - space with unit and $B^{+}$is $\|\bullet\|_{\gamma}-$ closed, solid, and pointed convex cone.

The space

$$
B C:=\left\{U \in B: U \text { is }\|\bullet\|-\text { continuous on } \ell_{\infty}^{+}\right\}
$$

is a closed subspace of $B$. However, this space is not a complete lattice. The corresponding positive pointed convex cone, denoted $(B C)^{+}$, is however normclosed and solid. Its weighted sup norm interior is nonempty since the weight function $\varphi_{\gamma} \in B C^{+}$is an order unit in this subspace of $B^{+}$with its induced partial order.

\subsection{The Koopmans Equation}

The aggregator approach to recovering recursive utility representations of an underlying preference relation defined on the given commodity space is expressed in terms of a functional equation. This equation takes the aggregator function as the primitive concept. The Koopmans equation for recursive utility is defined for each $C \in \ell_{\infty}^{+}$by the formula (where $S$ is the shift operator):

$$
U(C)=W\left(c_{1}, U(S C)\right)
$$

A solution of this equation is a recursive utility function representation of the preference relation. Of course, it all depends on what is meant by a solution. Proving this functional equation has a solution turns on recasting the problem as demonstrating a corresponding nonlinear operator, known as the Koopmans

\footnotetext{
${ }^{23}$ The norm $\|\bullet\|$ is a uniformly continuous real-valued function defined on the set $\ell_{\infty}$. See Aliprantis and Burkinshaw ([3], p. 218). Hence, the function $\varphi_{\gamma}(C)$ is continuous as the composition of the continuous functions $1+\|C\|$ and $\phi(x)=x^{1 / \gamma}$ for $x>0$.
} 
operator (denoted by $T_{W}$ ) has a fixed point in the desired function space of possible solutions. The Koopmans operator (defined below) is formally defined given a function $U \in B^{+}$by the following equation for each $C \in \ell_{\infty}^{+}$:

$$
\left(T_{W} U\right)(C)=W\left(c_{1}, U(S C)\right) .
$$

If $T_{W} U=U$, then $U$ is a solution to the Koopmans equation and defines a recursive representation of the underlying preference relation. That is, a fixed point is an element of $B^{+}$.

\subsection{Recovery Theory Review}

The Koopmans operator is readily shown to be a monotone self-map on $B^{+}$. The basic existence theory in Marinacci and Montrucchio [30], as extended by us, shows there are extremal solutions. There is a smallest fixed point, which is a norm lower semicontinuous function while the largest fixed point is a norm upper semicontinuous solution in the space $B^{+}$. Hence, if the Koopmans operator has a unique fixed point in $B^{+}$, then it must be norm continuous. That is, it is a function in $B C^{+} \subset B^{+}$. The existence of extremal fixed points is the stated as the Marinacci and Montrucchio [30] Recovery Theorem.

Theorem 7 Suppose $W$ is a concave Thompson aggregator. ${ }^{24}$

1. There is a $\|\bullet\|-$ upper semicontinuous function $U^{\infty} \in B^{+}$such that $T_{W} U^{\infty}=U^{\infty}$.

2. There is a $\|\bullet\|-$ lower semicontinuous function $U_{\infty} \in B^{+}$such that $T_{W} U_{\infty}=U_{\infty}$.

3. $U_{\infty}$ is the least fixed point, $U^{\infty}$ is the greatest fixed point, and the set of fixed points, fix $\left(T_{W}\right)$, is a countably chain complete subset of $B^{+} .{ }^{25}$

Our proof of the Recovery Theorem's is based on verifying the hypotheses of the Tarski-Kantorovich Fixed Point Theorem are met on an appropriately chosen order interval in the positive cone $B^{+}$. This order interval is denoted $\left\langle\theta, U^{T}\right\rangle$, where $\theta$ is the zero function in $B^{+}$and $U^{T}$, called " $U$-top," is defined below. Of course we require $U^{T} \in B^{+}$as well. Our proof verifies that the iterates of the Koopmans operator, denoted by $T_{W}^{N} U$ for each natural number and initial seed $U$ have the following properties: for $U=\theta:\left\{T_{W}^{N} \theta\right\} \nearrow U_{\infty}$; for $U=U^{T}:\left\{T_{W}^{N} U^{T}\right\} \searrow U^{\infty}$. That is, the least fixed point is approximated from below by the nondecreasing sequence $\left\{T_{W}^{N} \theta\right\}$ and the greatest fixed point is approximated from above by the nonincreasing sequence $\left\{T_{W}^{N} U^{T}\right\}$. The least fixed point is the principal solution of the Koopmans equation. ${ }^{26}$

\footnotetext{
${ }^{24}$ We state the result for our slightly more restrictive definition of the Thompson class. Our existence arguments apply to Marinacci and Montrucchio's [30] original axiomatic aggregator specification.

${ }^{25}$ Each countable chain in fix $\left(T_{w}\right)$ has an infimum and supremum in this set.

${ }^{26}$ Becker and Rincón-Zapatero [9] present economic, mathematical, and theoretical computation arguments for this designation.
} 
The desired order interval has the property $T_{W} \theta \geq \theta$ and $T_{W} U^{T} \leq U^{T}$ with $T_{W}:\left\langle\theta, U^{T}\right\rangle \rightarrow\left\langle\theta, U^{T}\right\rangle$. Evidently $T_{W} \theta \geq \theta$ since for each $C \in \ell_{\infty}^{+}$we have $T_{W} \theta(C)=W\left(c_{1}, 0\right) \geq 0$ as $\theta(S C)=0$. This order interval is a complete lattice in its induced order as a subset of $B^{+}$. Marinacci and Montrucchio [30] define $U^{T}$ in terms of the given aggregator and the weight function $\varphi_{\gamma}$ as follows:

$$
U^{T}(C)=W\left(1, y^{*}\right) \varphi_{\gamma}(C) .
$$

Here, the element $y^{*}>0$ is the unique solution to $W\left(1, y^{*}\right)=y^{*}$ (which exists since $W$ is a Thompson aggregator and $\alpha=1$ in (T6)). Evidently $U^{T} \in B^{+}$, $U^{T} \geq \theta, U^{T}(C)>0$ for each $C$, and $\left\|U^{T}\right\|_{\gamma}=W\left(1, y^{*}\right)<+\infty$. Furthermore, $U^{T} \in B C^{+}$follows from its definition.

Marinacci and Montrucchio [30] prove the Koopmans operator is a self-map on the order interval $\left\langle\theta, U^{T}\right\rangle$. The Recovery Theorem's extremal fixed points are elements of this order interval. Our aim is to show there is a unique solution in that order interval.

\subsection{Uniqueness Can Fail: A CES Example.}

The extremal fixed points may never be identical on the commodity space $\ell_{\infty}^{+}$. This may obtain whenever $W(0,0)=0$ holds, a common feature of the CES and KDW aggregators. Consider the CES aggregator (7) assuming $0<\rho<1$. Note there is a unique $y^{*}>0$ such that $W\left(1, y^{*}\right)=y^{*}$. Choose a natural number $N$. Compute $T_{W}^{N} \theta(C)$ and evaluate this expression at $C=0$ to obtain for each $N$ :

$$
T_{W}^{N} \theta(0)=W(0,0)=0 .
$$

Hence, passing to the limit we find $U_{\infty}(0)=0$. On the other hand, calculation of $U^{\infty}(0)$ proceeds as follows by computing the iterates directly for this CES aggregator:

$$
\begin{aligned}
T_{W}^{0} U^{T}(0) & \equiv U^{T}(0)=y^{*} \varphi_{\gamma}(0)=y^{*} \text { as } \varphi_{\gamma}(0)=1 ; \\
T_{W} U^{T}(0) & =W\left(0, y^{*}\right)=\beta\left(y^{*}\right)^{\rho} ; \\
T_{W}^{2} U^{T}(0) & =W\left(0, W\left(0, y^{*}\right)\right)=\beta\left[\beta\left(y^{*}\right)^{\rho}\right]^{\rho}=\beta^{1+\rho}\left(y^{*}\right)^{\rho^{2}} ; \\
& \vdots \\
T_{W}^{N} U^{T}(0) & =W\left(0, W\left(0, W\left(0, \ldots, W\left(0, y^{*}\right)=\beta^{\left(1+\rho+\rho^{2}+\cdots+\rho^{N-1}\right)}\left(y^{*}\right)^{\rho^{N}} .\right.\right.\right.
\end{aligned}
$$

Clearly $\rho^{N} \rightarrow 0$ as $N \rightarrow \infty$ implies $\lim _{N}\left(y^{*}\right)^{\rho^{N}}=1$ and

$$
U^{\infty}(0)=\lim _{N \rightarrow \infty} T_{W}^{N} U^{T}(0)=\beta^{\left(\frac{1}{1-\rho}\right)}>0,
$$

and hence, $U^{\infty}(0)>U_{\infty}(0)=0$. The extremal fixed points of the Koopmans operator cannot agree on the entire domain, $\ell_{\infty}^{+}$. A unique solution to the Koopmans equation is NOT uniquely determined by the aggregator function. 
Nonuniqueness problems can arise on the boundary of the consumption set. The origin is one such boundary point. There are counterexamples to uniqueness for other boundary consumption bundles. ${ }^{27}$ However, this does not mean we cannot say something useful about the subset of consumption sequences where the extremal fixed points deliver the same utility value. The papers by Marinacci and Montrucchio ([30],[31]), Martins-da Rocha and Vailakis [32] and Bloise and Vailakis [12], prove their uniqueness theorems by restricting the commodity space's domain for the utility functions. We do so here as well. Our common strategy is find uniqueness on the norm interior of $\ell_{\infty}^{+}$. We prove our uniqueness theorem first for the restricted commodity space where there is a minimum and maximum consumption constraint. These bounds are captured formally as restricting commodity bundles to an order interval in the positive cone's interior. The union of these sets turns out to cover the positive cone's norm interior. Details are given in the next section.

\section{Minimal and Maximal Consumption Sequences}

We follow the minimum and maximum consumption constraint technique appearing first in Martins-da Rocha and Vailakis [32]. The minimum consumption constraint is motivated in part by the observation that in conventional optimal growth models optimal consumption sequences are bounded away from zero at each time. Of course, this might NOT apply in models with exhaustible resources or where a renewable resource might bcome extinct as part of an optimal solution. A maximum consumption constraint is also placed on consumption sequences. This constraint is motivated by the presence of a maximum sustainable capital stock in the standard one-sector (or, two-sector) Ramsey optimal growth model with fixed labor. Aggregate capital and consumption are subject to diminishing marginal returns to capital investment. Of course, this rules out models capable of sustained growth such as the popular $A k$ - model where there is no labor constraint and the technology admits constant returns to capital inputs.

These minimal and maximal consumption constraints are formalized as order intervals which are bounded above and below in $\ell_{\infty}^{+}$. They have the specific form: $\langle a e, b e\rangle$ where $0<a<b<\infty$. The subset of $\ell_{\infty}^{+}$consisting of all sequences in this order interval is denoted by $K_{a b} \subset \ell_{\infty}^{+}$. Each $C \in K_{a b}$ satisfies $0<a \leq c_{t} \leq b$ for each $t$. Hence, $K_{a b} \subset \ell_{\infty}^{++}$as each sequence in $K_{a b}$ is an order unit in $\ell_{\infty}^{+}$. In our uniqueness theory we treat the numbers $a$ and $b$ as parameters that are varied (subject to $0<a<b<\infty$ ). The bounds on consumption sequences in $K_{a b}$ are critical in constructing bounds on aggregator values that are independent of any particular $C \in K_{a b}$. These bounds obtained

\footnotetext{
${ }^{27}$ Example 3 in Bloise and Vailakis [12] is instructive. They define the concave Thompson aggregator $W(x, y)=x+\min \{y, \alpha+\beta y\}$ where the parameters satisfy $\alpha>1$ and $0<\beta<1$. This aggregator's corresponding recursive utility function is not uniquely determined. Uniqueness fails for consumption sequences in the positive cone of $c_{0}$. These points of failure are necessarily boundary points in the larger commodity space's positive cone. Their uniqueness theory, and ours, exclude such consumption bundles.
} 
from the order interval $K_{a b}$ are fundamental to our adaptation of $u_{0}$ - concave operator theory to our problem.

It is reasonable to conjecture $\cup_{a, b} K_{a b}=\ell_{\infty}^{++}$. No element of $c_{0}^{+}$can belong to $K_{a b}$ for any $a>0$. Let $\mathcal{K}=\left\{K_{a b}\right\}$ for $0<a<b<\infty$ be the family of all order intervals of the form $K_{a b} \subset \ell_{\infty}^{++}$. This family covers $\ell_{\infty}^{++}$.

Lemma $8 \ell_{\infty}^{++}=\cup_{a, b} K_{a b}$.

Proof. Each $C \in \cup_{a, b} K_{a b}$ evidently belongs to some $K_{a, b}$ and must also be an order unit in $\ell_{\infty}^{+}$. Hence, $\cup_{a, b} K_{a b} \subseteq \ell_{\infty}^{++}$. The reverse containment follows immediately since $C \in \ell_{\infty}^{++}$is an order unit and belongs to $K_{a, b}$ with $0<a=$ $\inf _{t} c_{t}$ and some $b>a$ sufficiently large.

Our commodity space restriction is related to conditions imposed by Marinacci and Montrucchio [30] for their uniqueness theory. Specifically, they employ the following sets in their uniqueness theory:

$$
\begin{aligned}
C^{\gamma, 1} & =\left\{C \in \ell_{\infty}^{+}: W\left(c_{1}, 0\right)>0\right\} \\
A^{\gamma, 1} & =\left\{C \in \ell_{\infty}^{+}: \lim \sup _{t \rightarrow \infty} c_{t}<+\infty \& \lim \inf _{t \rightarrow \infty} W\left(c_{t}, 0\right)>0\right\} .
\end{aligned}
$$

The possibility that $\liminf c_{t}=0$ is excluded in the last set in many standard Thompson aggregators. For example, (joint) continuity of the aggregagor would imply $\lim _{t} W\left(c_{t}, 0\right)=0$ as $W(0,0)=0$ for the CES and KDW Thompson aggregators. ${ }^{28}$ However, if $\liminf _{t} c_{t}>0$, then in those same examples, $\liminf _{t} W\left(c_{t}, 0\right)>0$ holds as well. This implies for the CES and KDW aggregators that:

$$
\left\{C \in \ell_{\infty}^{++}: 0<\lim \inf _{t \rightarrow \infty} c_{t} \leq \lim \sup _{t \rightarrow \infty} c_{t}<+\infty\right\} \subset A^{\gamma, 1} .
$$

In fact $\inf _{t} c_{t} \leq \liminf _{t} c_{t}$ implies, by Lemma 8, that $\ell_{\infty}^{++} \subset A^{\gamma, 1}$. Hence, our commodity space restrictions may exclude some cases available in their theory. However, our approach is grounded on the more economically appealing abstraction motivating the minimal/maximum consumption sets $K_{a b}$. Our restrictions are modeled on the commodity space without interwining properties of the aggregator, as set forth in Marinacci and Montrucchio's [30] set $A^{\gamma, 1}$. We directly exclude the troublesome boundary points in the positive cone where we know (by examples) that uniqueness fails.

We adapt $u_{0}$ - concave operator theory to prove the Koopmans equation has a unique solution for possible utility functions defined on our restricted commodity space. We do this by choosing $u_{0}=T_{W} \theta \geq \theta, T_{W} \theta \neq \theta$ (i.e. $\left.T_{W} \theta>\theta\right)$. Then, we must verify that $T_{W}$ is $T_{W} \theta-$ concave. Motivated by Liang et al [28] we introduce the subset of $B^{+}$defined by

$B_{T_{W} \theta}^{+}=\left\{U \in B^{+}: \exists \alpha(U), \beta(U)>0\right.$ such that $\left.\alpha(U) T_{W} \theta \leq U \leq \beta(U) T_{W} \theta\right\}$.

\footnotetext{
${ }^{28}$ The Thompson properties $(T 1)-(T 4)$ do not formally require $W(0,0)=0$.
} 
Our goal is to show that the least fixed point $U_{\infty}$ is the unique solution to the Koopmans equation, at least among nonnegative $\varphi_{\gamma}$-bounded functions defined on $\ell_{\infty}^{++}$.

Our methods depend on the shift invariance of the order intervals in the collection $\left\{K_{a b}\right\}$ since we consider $U_{\infty} \mid K_{a b}$, the restriction of $U_{\infty}$ to the domain $K_{a b}$. We ask that $U_{\infty} \mid K_{a b}$ remain a solution on each such order interval. Hence, for a point $C \in K_{a b}$ we require

$$
U_{\infty}(C)=W\left(c_{1}, U_{\infty}(S C)\right)
$$

to hold. This equation only makes sense if $S C \in K_{a b}$ as well. This shift invariance property does NOT extend to order intervals built for growing economies. Let $\omega=\left(\alpha, \alpha^{2}, \alpha^{3}, \ldots\right)$ where the growth factor $\alpha>1$. Consider the order intervals of the form $\left\langle\frac{1}{n} e, n \omega\right\rangle \subset \ell_{\infty}^{+}(\alpha)$, the set of nonnegative real-valued sequences for which $\|C\|_{\alpha} \equiv \sup _{t}\left(c_{t} / \alpha^{t}\right)<+\infty$. The shift operator is not invariant on such an order interval. Set $n=1$ and consider $S \omega=\left(\alpha^{2}, \alpha^{3}, \alpha^{4}, \ldots\right)$. Then $\alpha^{2}>\alpha>1$ implies $S \omega \notin\langle e, \omega\rangle$.

Our methods cannot be improved to include consumption sequences that vanish at one, or more time periods. This follows from our counterexample (above) and the ones presented in Bloise and Vailakis [12]. The advantage of working with the minimal and maximum consumption requirement for sequences restricted to $K_{a b}$ is there are upper and lower bounds of the following type (by $W$ monotone and Thompson (T4)):

$$
\begin{aligned}
& \inf _{C \in K_{a b}} T_{W} \theta(C)=W(a, 0)>0 ; \\
& \sup _{C \in K_{a b}} T_{W} \theta(C)=W(b, 0)<\infty .
\end{aligned}
$$

The first equation above implies $T_{W} \theta>\theta$ holds for each $C \in K_{a b}$.

Let

$$
B^{+} \mid K_{a b}=\left\{U \in B^{+}: U \mid K_{a b} \rightarrow \mathbb{R}_{+} \text {and } U \geq \theta\right\} .
$$

This is just the set of trial utility functions in $B^{+}$restricted, or cut down, to the domain $K_{a b}$. The notation $U \mid K_{a b}$ is shorthand for $U: K_{a b} \rightarrow \mathbb{R}_{+}$and $U$ is nonnegative and $\varphi_{\gamma}$-bounded. Likewise, the set $B_{T_{W} \theta}^{+} \mid K_{a b}$ is just $B_{T_{W} \theta}^{+}$ restricted to functions defined on $K_{a b}$.

\section{$5 \quad T_{W}$ is $T_{W} \theta-$ Concave}

We prove that the Koopmans equation has at most one solution in the set of $\varphi_{\gamma}$-bounded functions defined on the set $\ell_{\infty}^{++}$by application of $u_{0}$ - concave operator theory. We verify Liang et al's [28] sufficient condition for the Koopmans operator to be a $T_{W} \theta$ - concave operator by showing this holds on the space of $\varphi_{\gamma}$-bounded nonnegative valued functions defined on each order interval $K_{a b}$. As the choices of the parameters $a$ an $b$ are arbitrary (other than $0<a<b<+\infty)$, we obtain uniqueness among the class of functions in $B^{+}$ whose domains are restricted to $\ell_{\infty}^{++}$. This function space is denoted $B^{+} \mid \ell_{\infty}^{++}$. 


\subsection{The Basic Ideas}

The first step is to prove the Koopmans operator is an ordered concave operator mapping from $B^{+} \mid K_{a b}$ to itself. Lemma 9 records this fact. The second step checks that the Koopmans operator actually sends points in $B^{+} \mid K_{a b}$ to $B_{T_{W} \theta}^{+} K_{a b}$. Lemma 10 reports this property. Liang et al's theorem applies to the cone $B^{+} \mid K_{a b}$ and Krasnosels'kii and Zabriecko's Theorem yields there is at most one nonzero fixed point in the positive cone $B^{+} \mid K_{a b}$.

Consider the Koopmans operator's least fixed point, $U_{\infty}$ in $\left\langle\theta, U^{T}\right\rangle \subset B^{+}$. It is sup norm lower semicontinuous. Moreover, $U_{\infty}(C) \geq T_{W} \theta(C) \geq \theta(C)=0$ for all $C \in \ell_{\infty}^{+}$and a strict inequality obtains for some $C$. Lemma 10 yields the restriction of the least fixed point to $K_{a b}$, denoted as $U_{\infty}\left|K_{a b} \in B_{T_{W} \theta}^{+}\right| K_{a b}$. Moreover, $U_{\infty} \mid K_{a b}$ must also solve the Koopmans equation on $B^{+} \mid K_{a b}$. The reason is simple: $T_{W} U_{\infty}(C)=U_{\infty}(C)=W\left(c_{1}, U_{\infty}(S C)\right)$ must hold for ALL $C \in \ell_{\infty}^{+}$, so a fortiori, this equality must also hold for each $C \in K_{a b}$. This observation makes use of the shift invariance property of $K_{a b}$. Note $U_{\infty}>\theta$ on each $K_{a b}$ since $W\left(a, U_{\infty}\left(S a_{c o n}\right)\right) \geq W(a, 0)>0$.

Thus if we can show there is at most one solution in $B^{+} \mid K_{a b}$, then $U_{\infty} \mid K_{a b}$ is the uniquely determined solution on that domain. This implies $U_{\infty}$ is the unique solution in $B^{+} \mid \ell_{\infty}^{++}$. The least and greatest fixed points agree on $\ell_{\infty}^{++}$.

\subsection{Uniqueness on Each $B^{+} \mid K_{a b}$.}

$B \mid K_{a b}$ is an $A M$-space with unit, $\varphi_{\gamma}$. Hence, the corresponding positive cone, $B^{+} \mid K_{a b}$, is a nonempty, $\|\bullet\|_{\gamma}-$ norm closed, pointed convex cone. $T_{W}$ is clearly a self map on $B^{+}$, consequently it is also a self map on the restricted space $B^{+} \mid K_{a b}$.

Lemma 9 establishes the monotone Koopmans operator is an ordered concave operator.

Lemma $9 T_{W}: B^{+}\left|K_{a b} \rightarrow B^{+}\right| K_{a b}$ and $T_{W}$ is an ordered-concave operator on $B^{+} \mid K_{a b}$.

Proof. That $T_{W}$ is an ordered concave operator follows from the concavity of the aggregator function and convexity of the cone $B^{+} \mid K_{a b}$. Fix an arbitrary $C \in K_{a b}$. Let $U^{0}, U^{1} \in B^{+} \mid K_{a b}$ and let $U^{t}=(1-t) U^{0}+t U^{1}$, for $0 \leq t \leq 1$. Evidently $U^{t} \in B^{+} \mid K_{a b}$. By $W$ concave in its second argument for each $c_{1}$,

$$
W\left(c_{1}, U^{t}(S C)\right) \geq(1-t) W\left(c_{1}, U^{0}(S C)\right)+t W\left(c_{1}, U^{1}(S C)\right) .
$$

The Lefthand side of this inequality is $T_{W} U^{t}(C)$ and the Righthand side is the convex combintation $(1-t) T_{W} U^{0}(C)+t T_{W} U^{1}(C)$. Thus,

$$
T_{W} U^{t} \geq(1-t) T_{W} U^{0}+t T_{W} U^{1}
$$

and $T_{W}$ is an ordered concave operator on $B^{+} \mid K_{a b} .{ }^{29}$

\footnotetext{
${ }^{29}$ Note that this proof does NOT require $U^{0} \geq U^{1}$ or $U^{1} \geq U^{0}$.
} 
Lemma 11 confirms the Koopmans operator's range is $B_{T_{W} \theta}^{+} \mid K_{a b}$. Corollary 12 states the Koopmans operator is $T_{W} \theta$-concave on $B^{+} \mid K_{a b}$. In Liang et al's [28] setup set $P \equiv B^{+} \mid K_{a b}$ and $u_{0} \equiv T_{W} \theta$. Cast $B_{T_{W} \theta}^{+} \mid K_{a b}$ in the role of $P_{u_{0}}$ as follows:

$$
B_{T_{W} \theta}^{+} \mid K_{a b}=\left\{\begin{array}{c}
V \in B^{+} \mid K_{a b}: \exists \alpha(V), \beta(V)>0 \\
\text { such that } \alpha(V) T_{W} \theta \leq V \leq \beta(V) T_{W} \theta
\end{array}\right\}
$$

Let $V \equiv T_{W} U \in B^{+} \mid K_{a b}$ for $U \in B^{+} \mid K_{a b}$. We must prove $V=T_{W} U \in$ $B_{T_{W} \theta}^{+} \mid K_{a b}$ as well.

Lemma $10 T_{W}^{+}: B^{+}\left|K_{a b} \rightarrow B_{T_{W} \theta}^{+}\right| K_{a b}$.

Proof. Since $T_{W}$ is a monotone operator, we always have $T_{W} U \geq T_{W} \theta$ whenever $U \geq \theta$. Hence, may set $\alpha(U)=1$ where we write the scalar $\alpha(V)=$ $\alpha\left(T_{W} U\right) \equiv \alpha(U)$ to simplify notation and note that this scalar ultimately depends only on $U$ and its image under $T_{W}$.

On the other hand, since $U$ is $\varphi_{\gamma}$ - bounded, there is a number $M^{U}$ such that $U(C) \leq M^{U} \varphi_{\gamma}(C)$ for each $C \in \ell_{\infty}^{+}$. Also note that $\varphi_{\gamma}(C) \leq \varphi_{\gamma}(b e)=$ $(1+b)^{1 / \gamma}$ for each $C \in K_{a b}$. Thus, by monotonicity of the Koopmans operator we obtain for each $C \in K_{a b}$ :

$$
T_{W} U(C) \leq T_{W} \varphi_{\gamma}(C) \leq W\left(c_{1}, M^{U}(1+b)^{1 / \gamma}\right) .
$$

Moreover, $T_{W} \theta(C)=W\left(c_{1}, 0\right) \geq W(a, 0)$ by monotonicity of the aggregator function and the definition of $K_{a b}$. Since $W(a, 0)>0$ by Thompson property $(T 4)$, we can choose a number $\beta(U)$ large enough such that

$$
W\left(c_{1}, M^{U}(1+b)^{1 / \gamma}\right) \leq \beta(U) W(a, 0) .
$$

Then note that $\beta(U) W(a, 0) \leq \beta(U) T_{W} \theta(C)$ and the previous inequality yields for each $C \in K_{a b}$ :

$$
T_{W} U(C) \leq \beta(U) T_{W} \theta(C) .
$$

Thus, for $\alpha(U)=1$, and this choice of $\beta(U)$, we find

$$
T_{W} \theta \leq T_{W} U \leq \beta(U) T_{W} \theta .
$$

This proves $T_{W} U \in B_{T_{W} \theta}^{+} \mid K_{a b}$.

Inequality (10) is readily rearranged in the form of inequality (5): for $\mu(U)=$ $1 / \beta(U)>0$ :

$$
\mu(U) T_{W} U \leq T_{W} \theta .
$$

Combining the previous two lemmas yields:

Corollary $11 T_{W}: B^{+}\left|K_{a b} \rightarrow B_{T_{W} \theta}^{+}\right| K_{a b}$ is a $T_{W} \theta-$ concave operator for each $0<a<b<\infty$. 
Proof. Lemmas 9 and 10 imply the Koopmans operator satisfies the hypotheses of Liang et al's Theorem. Hence $T_{W}$ is a $T_{W} \theta-$ concave operator acting on each cone $B^{+} \mid K_{a b}$.

Finally, we invoke the Krasnosel'skiı and Zabreiko Theorem when the Koopmans operator acts on $B^{+} \mid K_{a b}$.

Proposition 12 For each given $0<a<b<\infty$, the Koopmans equation, $T_{W} U=U$, has at most one non-zero solution in the cone $B^{+} \mid K_{a b}$.

The nonzero principal fixed point $U_{\infty} \in B^{+}$remains a nonzero solution to the Koopmans equation on the domain $B^{+} \mid K_{a b}$. Hence, Proposition 13 implies

$$
T_{W} U_{\infty}\left|K_{a b}=U_{\infty}\right| K_{a b}
$$

for each $0<a<b<\infty$ is the unique solution in $B^{+} \mid K_{a b}$. Here, the order interval $K_{a b}$ is arbitrarily chosen. Therefore, we conclude $U_{\infty}$ is the unique solution among all the functions in $B^{+} \mid \ell_{\infty}^{++}$.

Uniqueness on $\ell_{\infty}^{++}$implies $U^{\infty}=U_{\infty}$ agree on that domain. $U_{\infty}$ is sup norm lower semicontinuous and $U^{\infty}$ is sup norm upper semicontinuous. Hence, the operator's principal solution is also a sup norm continuous function on $\ell_{\infty}^{++}$.

We sum up our findings:

Theorem 13 Let $W$ be a concave Thompson aggregator. Then, there is a unique nonzero norm continuous utility function $U_{\infty} \in B^{+} \mid \ell_{\infty}^{++}$that solves the Koopmans equation. That is, for each $C \in \ell_{\infty}^{++}$:

$$
T_{W} U_{\infty}(C)=U_{\infty}(C)=W\left(c_{1}, U_{\infty}(S C)\right) .
$$

\section{Concluding Comments}

Our Theorem says the recursive utility function is uniquely determined by the aggregator at each consumption sequence in the interior of the commodity space's positive cone. We must exclude null consumption sequences as well as all other consumption sequences in the positive cone's boundary. These restrictions are the same as the ones imposed in Martins-da-Rocha and Vailakis [32], Bloise and Vailakis [12] and Marinacci and Montrucchio [31]. They are formally more restrictive than constraints imposed by Marinacci and Montrucchio [30] who exploit properties of the aggregator in defining their commodity space restrictions. We strengthen Marinacci and Montrucchio's [30] Thompson aggregatgor definition to include joint continuity and concavity of the aggregator on its domain. The joint concavity assumption is stronger than their assumption that the aggregator is concave in its second argument at zero. We strengthen their conditions in order to prove the Koopmans operator is an ordered concave operator and verify it is also a $u_{0}$-concave operator by Liang, et al's [28] Theorem. This approach differs from the methods used in the existing literature. The concave operator techique is an alternative for demonstrating 
uniqueness results compared to the contraction operator theorems based on the Thompson metric employed by Marinacci and Montrucchio ([30], [31]) or the 0-local contractions introduced by Rincón-Zapatero and Rodriguez-Palmero ([36], [37]) and Martins-da-Rocha and Vailakis ([32], [33]). It is worth noting the uniqueness contribution by Marinacci and Montrucchio [30] accommodates some endogenous growth models, whereas we are not able to do so.

The $u_{0}$ - concave operator theory required one important economic restriction. The underlying commodity space could NOT admit sustainable growth or balanced growth paths. It would be of some interest to work out a concave operator based uniqueness theory for the norm interiors of the weighted commodity spaces that model sustainable growth. That is, find a uniqueness theory compatible with the general existence theory for solutions to the Koopmans equation when the economic model includes a variety of endogenous growth theories. 


\section{References}

[1] Charalambos D. Aliprantis and Kim C. Border, Infinite Dimensional Analysis: A Hitchhiker's Guide, $3^{\text {rd }}$-Edition, Springer, New York, 2006.

[2] Charalambos D. Aliprantis and Own Burkinshaw, Positive Operators, Academic Press, San Diego, 1985.

[3] Charalambos D. Aliprantis and Own Burkinshaw, Principles of Real Analysis, $3^{\text {rd }}$-Edition, Academic Press, San Diego, 1998.

[4] Charalambos D. Aliprantis and Rabee Tourky, Cones and Duality, American Mathematical Society, Providence, 2007.

[5] Łukasz Balbus, Kevin Reffett, and Łukasz Woźny, "Time Consistent Markov Policies in Dynamic Economies with Quasi-Hyperbolic Consumers," International Journal of Game Theory, 44, 2015, pp. 83-112.

[6] Robert A. Becker and John H. Boyd III, Capital Theory, Equilibrium Analysis, and Recursive Utility, Blackwell Publishing, 1997.

[7] Robert A. Becker, John H. Boyd, III, and Ciprian Foias, "The Existence of Ramsey Equilibrium," Econometrica, Vol. 59 (2), March 1991, pp. 441-460.

[8] Robert A. Becker and Juan Pablo Rincón-Zapatero, "Recursive Utility and Thompson Aggregators," Indiana University CAEPR Working Paper \#XX, July 22, 2017 (revised August 29, 2017).

[9] Robert A. Becker and Juan Pablo Rincón-Zapatero, "Recursive Utility and Thompson Aggregators I: Constructive Existence Theory for the Koopmans Equation," Indiana University CAEPR Working Paper, July 12, 2018.

[10] Garrett Birkhoff, Lattice Theory, American Mathematical Society, Providence, RI, 1940.

[11] David Blackwell, "Discounted Dynamic Programming," Annals of Mathematical Statistics, 36, 1965, pp. 226-235.

[12] Gaetano Bloise and Yiannis Vailakis, "Convex Dynamic Programming with (Bounded) Recursive Utility," Journal of Economic Theory, 173, 2018, pp. 118-141.

[13] John H. Boyd III, "Recursive Utility and the Ramsey Problem," Journal of Economic Theory, 50, 1990, pp. 326-345.

[14] Wilbur John Coleman, "Equilibrium in a Production Economy with an Income Tax," Econometrica, Vol. 59(4), July 1991, pp. 1091-1104.

[15] B.A. Davey and J.A. Priestley, Introduction to Lattices and Order, Cambridge University Press, Cambridge, UK, 1990. 
[16] Dajun Guo and V. Kakshmikantham, Nonlinear Problems in Abstract Cones, Academic Press, San Diego, 1988.

[17] Dajun Guo, Yeol Je Cho, and Jiang Zhu, Partial Ordering Methods in Nonlinear Problems, Nova Science Publishers, New York, 2004.

[18] Manjira Datta and Kevin L. Reffett, "Isotone Recursive Methods: The Case of Homogeneous Agents," in Handbook on Optimal Growth 1: Discrete Time (Rose-Anne Dana, Cuong Le Van, Tapan Mitra, and Kazuo Nishimura, eds.), Springer-Verlag, Berlin, 2006.

[19] John L. Kelley, General Topology, Van Nostrand Reinhold Co., New York, 1955.

[20] Tjalling C. Koopmans, "Stationary Ordinal Utility and Impatience," Econometrica, 28, 1960, 287-309.

[21] Tjalling C. Koopmans, "Representation of Preference Orderings Over Time," in Decision and Organization (CB. McGuire and Roy Radner, eds.), North-Holland, Amsterdam, 1972.

[22] Tjalling C. Koopmans, "Representation of Preference Orderings with Independent Components of Consumption,"in Decision and Organization (CB. McGuire and Roy Radner, eds.), North-Holland, Amsterdam, 1972.

[23] Tjalling C. Koopmans, Peter A. Diamond, and Richard E. Williamson, "Stationary Utility and Time Perspective," Econometrica, 82, 1964, pp. $82-100$.

[24] M.A. Krasnosel'skiı̌, Positive Solutions of Operator Equations, P. Noordhoff LTD, Groningen, The Netherlands, 1964.

[25] M.A. Krasnosel'skiı̌ and P.P. Zabreı̌ko, Geometrical Methods of Nonlinear Analysis, Springer-Verlag, Berlin, 1984.

[26] Cuong Le Van, Lisa Morhaim, Yiannis Vailakis, "Monotone Concave Operators: An Application to the Existence and Uniqueness of Solutions to the Bellman Equation," Working Paper July 2008 HAL Id: < hal-00294828>.

[27] Coung Le Van and Yiannis Vailakis, "Recursive Utility and Optimal Growth with Bounded or Unbounded Returns," Journal of Economic Theory, 123, 2005, 187-209.

[28] Zhan Dong Liang, Wen Xia Wang, and Sheng Jia Li, "On Concave Operators," Acta Mathematica Sinica, English Series, March 2006, Vol. 22 (2), pp. $577-582$.

[29] Robert E. Lucas and Nancy L. Stokey, "Optimal Growth with Many Consumers," Journal of Economic Theory, 32, 1984, pp. 139-171. 
[30] Massimo Marinacci and Luigi Montrucchio, "Unique Solutions for Stochastic Recursive Utilities," Journal of Economic Theory, 145, 2010, pp. 17761804.

[31] Massimo Marinacci and Luigi Montrucchio, "Unique Tarski Fixed Points," Working Paper No. 604, University of Bocconi, July 2017.

[32] V. Felipe Martins-da-Rocha and Yiannis Vailakis, "Existence and Uniqueness of a Fixed Point for Local Contractions," Econometrica, Pol. 78 (3), May 2010, pp. 1127-1141.

[33] V. Felipe Martins-da-Rocha and Yiannis Vailakis, "Fixed Point for Local Contractions: Applications to Recursive Utility," International Journal of Economic Theory, 9, 2013, pp. 23-33.

[34] Peter Meyer-Nieberg, Banach Lattices, Springer-Verlag, New York, 1991.

[35] Anthony L. Peressini, Ordered Topological Vector Spaces, Harper \& Row, New York, 1967.

[36] Juan Pablo Rincón-Zapatero and Carlos Rodriguez-Palmero, "Existence and Uniqueness to the Bellman Equation in the Unbounded Case," Econometrica, 71 (5), September 2003, pp. 1519-1555.

[37] Juan Pablo Rincón-Zapatero and Carlos Rodriguez-Palmero, "Recursive Utility with Unbounded Aggregators," Economic Theory, 33, 2007, pp. 381-391.

[38] A. Tarski, "A Lattice-Theoretical Fixpoint Theorem and Applications," Pacific Journal of Mathematics, 3, 1955, pp. 285-309.

[39] B.Z. Vulikh, Introduction to the Theory of Partially Ordered Spaces, Wolters-Noordhoff Scientific Publications, Gronigen, Netherlands, 1979. 